\title{
Complex analysis of pre-seismic geoacoustic and electromagnetic emission signals
}

\author{
Olga Lukovenkova ${ }^{1, *}$, Alexandra Solodchuk ${ }^{1}$, Alexander Tristanov ${ }^{1}$, and Evgeniy Malkin ${ }^{2}$ \\ ${ }^{1}$ Institute of Cosmophysical Research and Radio Wave Propagation, Far Eastern Branch of the \\ Russian Academy of Sciences, Acoustic Researches Laboratory, Russia, 684034 Kamchatka region, \\ Elizovskiy district, Paratunka, Mirnaya str., 7 \\ ${ }^{2}$ Institute of Cosmophysical Research and Radio Wave Propagation, Far Eastern Branch of the \\ Russian Academy of Sciences, Electromagnetic Radiation Laboratory, Russia, 684034 Kamchatka \\ region, Elizovskiy district, Paratunka, Mirnaya str., 7
}

\begin{abstract}
The article describes the results of complex analysis of preseismic signals of electromagnetic and geoacoustic radiation. We analyzed the frequency content of single sferics and geoacoustic impulses recorded before the Zhupanov earthquake that occurred on January 30, 2016. The signals were analyzed using sparse approximation method, in particular Adaptive Matched Pursuit. Background signals were studied together with pre-seismic ones. Distributions of frequencies, that are part of background and pre-seismic signals, were compared. Differences in the frequency content of pre-seismic sferics and geoacoustic impulses were found. The revealed features of pre-seismic signals in the future can be used in the design of systems for monitoring, forecasting and prevention of natural disasters. The research was supported by Russian Science Foundation (project No. 18-11-00087).
\end{abstract}

\section{Introduction}

The results of acoustic and electromagnetic field complex studies in the frequency range from $200 \mathrm{~Hz}$ to $11 \mathrm{kHz}$ conducted in Kamchatka in 2013 show the presence of simultaneous anomalous bursts in electromagnetic and geoacoustic emission before earthquakes with magnitudes $\mathrm{M}_{\mathrm{LH}} \geq 5$ at epicentral distances up to $200 \mathrm{~km}$ [1]. According to the authors, the common cause of these anomalies is the activation of deformation processes during the preparation of an earthquake. Anomalies in the considered fields are detected in the azimuthal distribution of the lightning discharges number during the day (Fig. 1a) and in the amplitude variations of electromagnetic and geoacoustic emission envelope (Fig. 1b,c) [1-4]. Electromagnetic emission is estimated at eleven frequencies. The frequency of $1709 \mathrm{~Hz}$ is the resonant frequency of the Earth-ionosphere waveguide.

\footnotetext{
* Corresponding author: o.o.lukovenkova@yandex.ru
} 


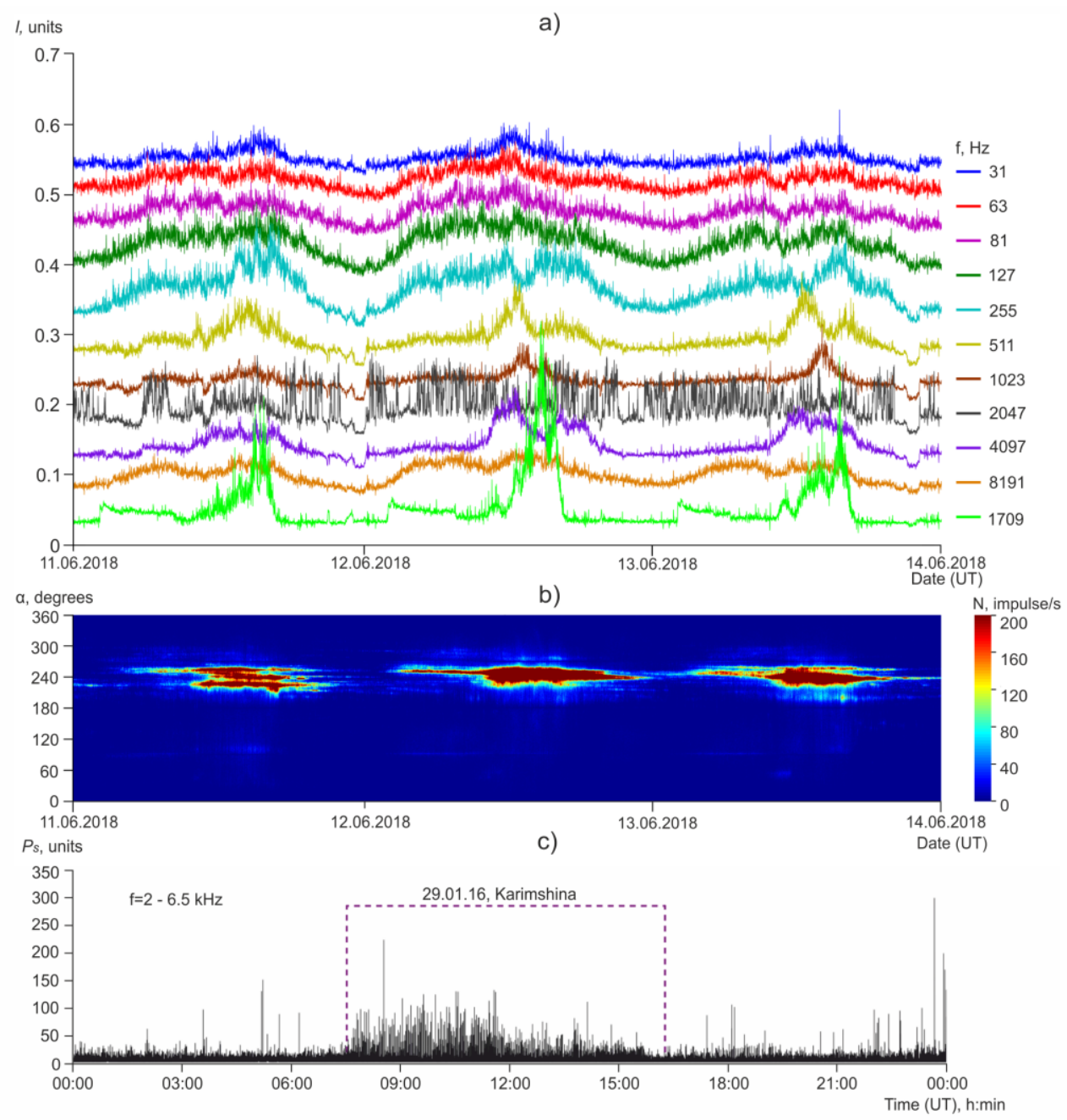

Fig. 1. Examples of anomalies: amplitude variations of electromagnetic emission envelope $(a)$; azimuthal distribution of electromagnetic emission impulses $(b)$; amplitude variations of geoacoustic emission envelope $(c)$

Continuous monitoring of the studied signals is carried out in the "Karymshina" site $\left(52.82^{\circ} \mathrm{N}, 158.13^{\circ} \mathrm{E}\right)$. Electromagnetic signals are observed using a multi-channel VLF recorder that receives weak VLF noise signals. The recorder consists of three antennas, preamplifiers, filtering device and analog-to-digital converter [3]. Geoacoustic signals are observed using piezoceramic receivers located at the bottom of artificial reservoirs [5]. Sampling frequency of both types of signals is from $44.1 \mathrm{kHz}$ to $48 \mathrm{kHz}$.

At the current stage of the research, simultaneous anomalies are detected in the integral characteristics of electromagnetic and geoacoustic signals (emission envelopes), not in the "live" data. The authors decided to analyze in detail the signal internal structure and to find out if described anomalies are accompanied by changes in the internal structure of the signals registered at the same time.

Both electromagnetic and geoacoustic signals have a pulsed nature. The signal parts containing impulses are of the greatest interest for researchers. A single impulse of natural 
origin electromagnetic emission propagating in the Earth-ionosphere waveguide called "sferic", and a single impulse of geoacoustic emission called "geoacoustic impulse".

The present work is devoted to the analysis of time- frequency structure of the sferics and the geoacoustic impulses recorded on before the Zhupanov earthquake occurred on January 30, 2016 at 03:25 UTC in Kamchatka (energy class Ks is 15.7; epicenter coordinates are $53.85^{\circ} \mathrm{N}, 159.03^{\circ} \mathrm{E}$; depth is $185 \mathrm{~km}$; epicenter distance is $126 \mathrm{~km}$; azimuth is $30^{\circ}$ relative to the "Karymshina" site; information about the earthquake is provided by the KB GS RAS). The seismic energy released during the Zhupanov earthquake was $5 \cdot 10^{15} \mathrm{~J}$. It allows us to consider this earthquake as one of the most powerful seismic events for all time of detailed observations in Kamchatka [6]. Earthquakes with energy class $K_{s}>15.0$ occur in Kamchatka every 5-10 years.

In [6] it is stated that a successful prediction was given on January 28, 2016 and the decision was made according to observation of VLF electromagnetic emission. According to observation data from "Karymshina" site anomalous bursts were also detected in the variations of the acoustic pressure accumulated on the one-second interval in the frequency range from 2 to $6.5 \mathrm{kHz}$ on January 29, 2016.

600 pre-seismic and 740 background sferics registered on January 22, 2016, also 3095 single geoacoustic impulses recorded on January 29, 2016 during the anomalous bursts of the accumulated acoustic pressure and 4044 geoacoustic impulses registered in the quiet periods on January 22, 2016 were selected for complex analysis.

\section{Complex analysis methods}

The authors have repeatedly shown the effectiveness of the sparse approximation method for the analysis and modeling of electromagnetic and geoacoustic pulsed signals in earlier studies [5,7-9]. Therefore, Adaptive Matching Pursuit (AMP) was chosen to study the timefrequency structure of sferics and geoacoustic impulses [5]. The general approach of different-nature pulsed signals analysis using sparse approximation method and the features of application AMP to sferics and geoacoustic impulses are described in [10].

Since various interferences are present at frequencies above $11 \mathrm{kHz}$ in the electromagnetic and geoacoustic signals and the frequency range of anomaly detection is limited to $11 \mathrm{kHz}$, the signals were pre-processed (filtered). Then filtered signals were represented as a linear combination of basis functions using the AMP algorithm. Each function described by a set of parameters such as frequency, time shift, duration, amplitude, and coefficients affecting the envelope shape.

The authors are most interested in the frequency content of the analyzed signals, so the search for anomalies was primarily carried out in the distributions of frequencies of the basis functions that consisting the impulses.

The technique of complex analysis can be briefly described as:

1. Preprocessing. It is proposed to filter signals using low-pass Butterworth filter with a cutoff frequency of $11 \mathrm{kHz}$.

2. Synthesis of function dictionary for sparse approximation. The signals are divided into groups according to their duration. The investigated sferics and geoacoustic impulses were divided into 4 groups: up to 200 samples, 200-500 samples, 500-1000 samples, more than 1000 samples. For each group a dictionary of basis functions with different maximum duration of functions (200, 500, 1000 and 3000 samples, respectively) and with following parameters was synthesized:

- $\quad$ type of function is Berlage;

- $\quad$ frequency $f$ is from 200 to $10000 \mathrm{~Hz}$;

- $\quad$ function duration (percentage of maximum possible duration) $p_{\text {end }}$ is from 0.15 to 1 ; 
- $\quad$ maximum position $p_{\max }$ is from 0.05 to 0.3 ;

- $\quad$ coefficient of envelope steepness $\Delta$ is from 1.1 to 5 .

3. Analysis. Signal analysis is carried out using AMP algorithm (maximum error is $5 \%$, count of refinement iterations is 7 , refinement accuracy is $5 \mathrm{~Hz}, 0.2,0.2,0.1$ for parameters $f, p_{\text {end, }} p_{\text {max }}, \Delta$ respectively).

4. Statistical processing of the analysis results. Following characteristics are determined separately for background and pre-seismic impulses:

- $\quad$ average signal duration;

- $\quad$ average signal amplitude;

- $\quad$ average sparsity of the resulting representations;

- estimate of the distribution of frequencies of the highest amplitude basis function;

- $\quad$ estimate of the distribution of frequencies of the first two basis functions with the highest amplitude;

- $\quad$ estimate of the all frequencies distribution.

5. Comparison of background and pre-seismic signal analysis results. The distribution estimates are compared. The similarities and differences in the background and preseismic signals frequency content are determined.

\section{Results of complex analysis}

\subsection{Electromagnetic emission}

Computational experiment performed by the authors was devoted to the analysis of timefrequency structure of the pre-seismic sferics. Data for the experiment and signs of anomalies are provided by the Laboratory of Electromagnetic Radiation IKIR FEB RAS. Despite the fact that the short-term and operational forecasting of strong earthquakes is possible, further improvement of the methods of measurement and signal processing is needed to clarify the features of pre-seismic anomalies of electromagnetic signals [1]. The results of analysis of the sferics internal structure are described below.

600 signals recorded on January 22, 2016 before the Zhupanov earthquake during periods of abnormal activity of electromagnetic emission were analyzed according methods that that have been described in paragraph 2. To identify the features of anomalous signal structure the time-frequency structure of pre-seismic sferics compared with the structure of typical sferics recorded on January 22, 2016 during quiet periods. 740 background sferics were processed and analyzed in the same way as anomalous ones.

Frequencies below $1500-2000 \mathrm{~Hz}$ should not be in the sferics due to the peculiarities of signal propagation in the Earth-ionosphere waveguide (the critical frequency of the waveguide). But frequencies from 0 to $1000 \mathrm{~Hz}$ may be in the near sferics. Thus, the timefrequency structure of typical sferic does not contain frequencies from the range $\sim 1000$ $2000 \mathrm{~Hz}$ (the range may be shifted due to different interference). All sferics having the time-frequency structure different from the declared one can be considered as anomalies.

The distribution of frequencies of the functions included in the signals was constructed for both groups of the signals. Fig. $2 a-c$ shows the distribution estimates built by the Kernel Density Estimate method (KDE) and the histogram method. Table 1 shows the main characteristics of signals and their sparse representations. 


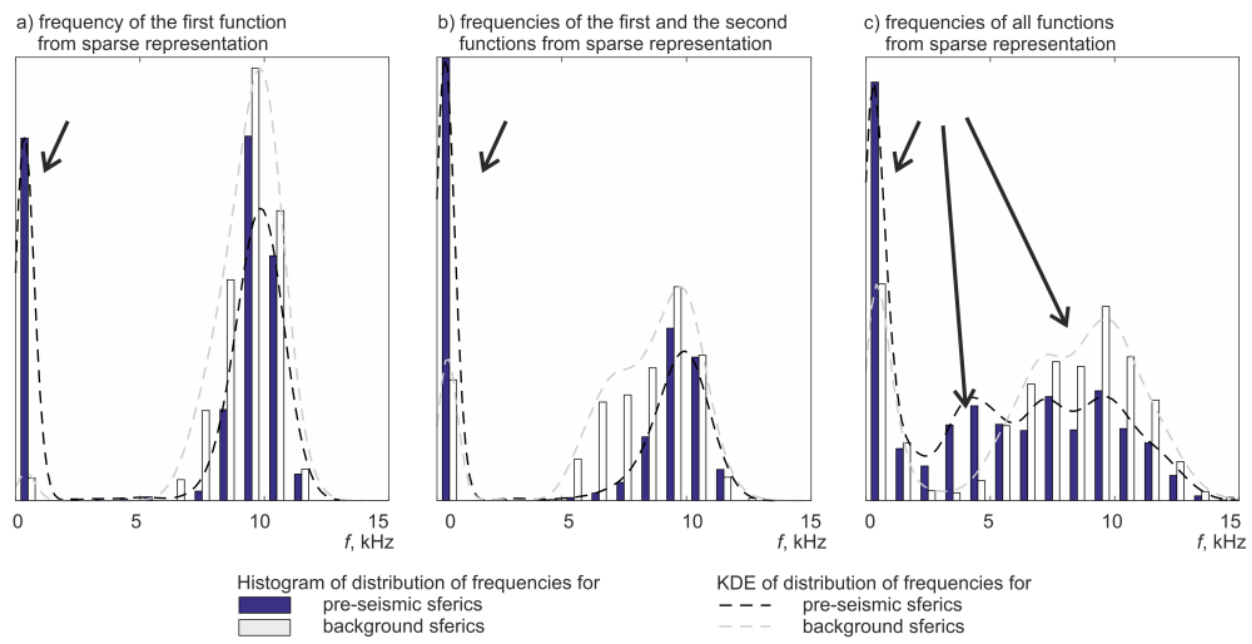

Fig. 2. The distributions of frequencies for pre-seismic and background sferics: by the basis function with the highest amplitude (a); by first two basis functions with the highest amplitude $(b)$; by all basis functions $(c)$. The arrows show differences in the frequency content.

Table 1. Characteristics of pre-seismic and background sferics.

\begin{tabular}{|l|l|l|}
\hline \multicolumn{1}{|c|}{ Characteristic } & \multicolumn{1}{|c|}{ Pre-seismic signals } & \multicolumn{1}{c|}{ Background signals } \\
\hline Average signal duration & 304 samples & 113 samples \\
\hline Average signal amplitude & 0.081 units & 0.0207 units \\
\hline $\begin{array}{l}\text { Average sparsity of the resulting } \\
\text { representations }\end{array}$ & 8 functions & 6 functions \\
\hline
\end{tabular}

Signal representation analysis shows that anomalous pre-seismic sferics have a longer duration, a higher amplitude and a more complex structure. The pre-seismic sferics have some differences in the frequency content. The most obvious differences are found in the estimate of the all frequencies distribution (Fig. 2c), i.e. changes are observed in the detailing basis functions having small amplitude:

- $\quad$ pronounced low-frequency component from 0 to $1 \mathrm{kHz}$;

- $\quad$ presence of frequencies in the range from 2 to $5 \mathrm{kHz}$;

- $\quad$ decrease in the frequency range from 5 to $7 \mathrm{kHz}$.

The first difference may indicate that the pre-seismic sferics passed a distance smaller than the background signals. The second and third ones may be due to areas with high electron concentration at the edges of the Earth-ionosphere waveguide [11].

\subsection{Geoacoustic emission}

Geoacoustic emission signals were processed together with sferics. 3095 pre-seismic and 4044 background impulses were selected for this experiment. It should be noted that the presence of clear criteria for typical sferics allowed to divide the signals into anomalous and background ones manually. Such criteria have not yet been developed for geoacoustic signals due to the wide variety of impulse forms. So the classification of geoacoustic impulses was carried out by the time of registration. The impulses registered during the periods of acoustic pressure bursts on January 29, 2016 were considered as pre-seismic 
signals. The impulses registered during the quiet periods on January 22, 2016 were considered as background ones. All signals were analyzed according to the methods described in paragraph 2.

Fig. $3 a-c$ shows estimates of the frequency distributions of the functions included in the sparse signal representations. Table 2 shows the main characteristics of pre-seismic and background geoacoustic pulses.
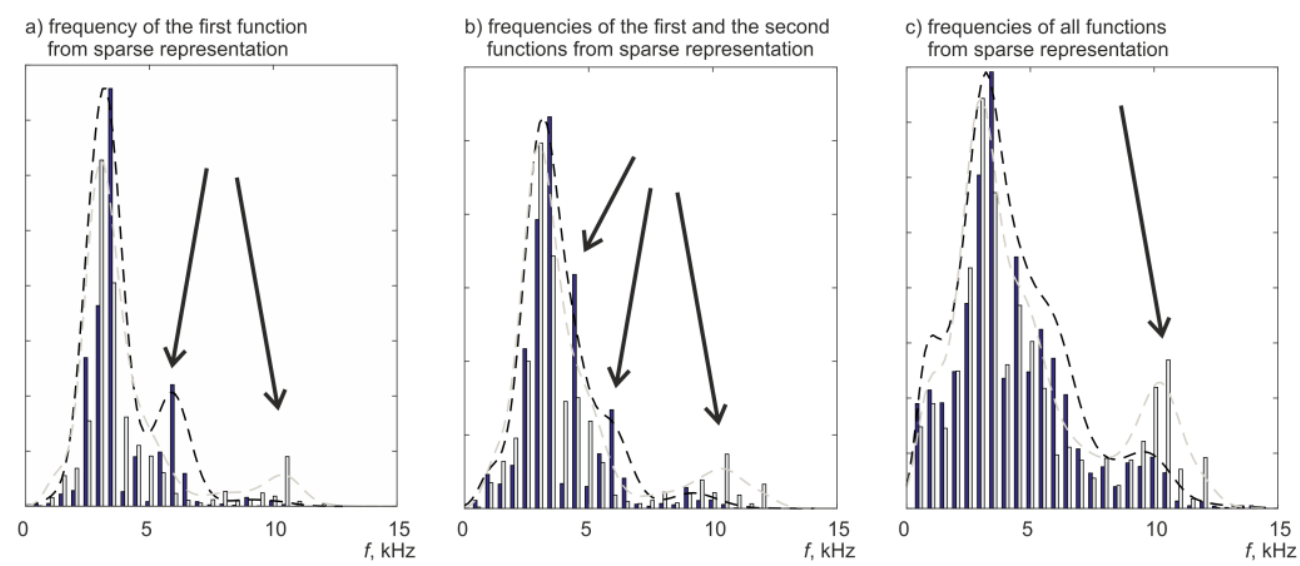

Histogram of distribution of frequencies for
pre-seismic geoacoustic impulses

$\mathrm{KDE}$ of distribution of frequencies for

- - $\quad$ pre-seismic geoacoustic impulses

Fig. 3. The distributions of frequencies for pre-seismic and background geoacoustic impulses: by the basis function with the highest amplitude $(a)$; by first two basis functions with the highest amplitude $(b)$; by all basis functions $(c)$. The arrows show differences in the frequency content.

Table 2. Characteristics of pre-seismic and background sferics.

\begin{tabular}{|l|l|l|}
\hline \multicolumn{1}{|c|}{ Characteristic } & \multicolumn{1}{|c|}{ Pre-seismic signals } & \multicolumn{1}{|c|}{ Background signals } \\
\hline Average signal duration & 487 samples & 577 samples \\
\hline Average signal amplitude & 0.1717 units & 0.1705 units \\
\hline $\begin{array}{l}\text { Average sparsity of the resulting } \\
\text { representations }\end{array}$ & 7 functions & 9 functions \\
\hline
\end{tabular}

According to Table II it can be concluded that there are no obvious differences in the form and duration of background and pre-seismic geoacoustic impulses. There are short (up to $5 \mathrm{~ms}$ ) and long (15-30 ms) impulses and impulses with small and high amplitudes in both groups. So unlike electromagnetic signals pre-seismic geoacoustic signals are not visually different from the background ones.

There are differences in the frequency content of the pre-seismic and background signals (Fig. 3a-c). But these differences are not as obvious as ones in electromagnetic signals. Also, they are detected in the distributions of the frequencies of first two functions with the highest amplitude included in the sparse representation of signal:

- $\quad$ there are peaks in the region of $4 \mathrm{kHz}$ (Fig. $3 b$ ) and $6 \mathrm{kHz}$ (Fig. 3a);

- pre-seismic signals have less pronounced high-frequency component (more than $10 \mathrm{kHz}$ ).

There is no physical explanation for these features of pre-seismic geoacoustic impulses. The authors suggest that the increase in the number of impulses with some frequency (in our 
case 4 and $6 \mathrm{kHz}$ ) may be due to the activation of geoacoustic emission in some directions before the earthquake.

\section{Results}

The complex analysis of geoacoustic and electromagnetic emission signals registered before the Zhupanov earthquake revealed that their time-frequency structure differs from the structure of background signals.

There were obvious differences in the frequency content of the pre-seismic sferics: the low-frequency component (up to $1 \mathrm{kHz}$ ) is significantly expressed and frequencies in the range from 2 to $5 \mathrm{kHz}$ are present. In the background sferics, as a rule, a dip is observed in the range from 2 to $5 \mathrm{kHz}$, which is explained by features of their propagation in the Earthionosphere waveguide. In general, anomalous sferics have a longer duration and a more complex time-frequency structure.

The analysis of geoacoustic signals is complicated by several reasons. Firstly, a wide variety of impulses associated with the superposition of signals from multiple sources at the receiving point and with the influence of possible noise of technogenic and natural origin. Secondly, in contrast to sferics, the natural environment model (sedimentary rocks), in which the geoacoustic signals are generated, is currently not fully formed due to its complexity.

The analysis of the temporal forms of pre-seismic signals revealed no differences either in form or in duration. As a result of the analysis of the frequency content of pre-seismic and background impulses, it was determined that an increase in the number of impulses with frequencies of 4 and $6 \mathrm{kHz}$ occurred. It was observed during periods of anomalies in the amplitude variations of the geoacoustic emission envelope before the Zhupanov earthquake.

In the future, to identify the characteristic features of anomalous pre-seismic signals, it is planned to conduct a study of sferics and geoacoustic impulses preceding other seismic events in Kamchatka.

\section{References}

1. G.I. Druzhin, Iu.V. Marapulets, N.V. Cherneva et al., Doklady Earth sciences, 472(5), 584 (2017)

2. G.I. Druzhin,Volcanology \& Seismology, 6, 51 (2002)

3. I.A. Larionov, E.I. Malkin, Iu.V. Marapulets et al. Bulletin KRASEC. Physical and Mathematical Sciences, 24(4), 213 (2018)

4. Y.V. Marapulets, B.M. Shevtsov, I.A. Larionov et al., Russian Journal of Pacific Geology, 6(6), 457, (2012)

5. Y.V. Marapulets, O.O. Lukovenkova, A.B. Tristanov et al., Methods for recording and for time-frequency analysis of geoacoustic emission signals (Dalnauka, Vladivostok, 2017)

6. V.N. Chebrov, Iu.A. Kugaenko, I.R. Abubakirov et al., Bulletin KRASEC. Earth sciences, 29(1), 5 (2016)

7. Yu.I. Senkevich, O.O. Lukovenkova, A.A. Solodchuk et al., Geosystems of Transition Zones, 4(2), 409 (2018)

8. M.I. Gapeev, Yu.I. Senkevich, O.O. Lukovenkova et al., Proc. of ITNT-2019, 455 (2019)

9. O. Lukovenkova, Y. Marapulets, A. Tristanov et al., E3S Web of Conferences, (2018)

10. O.O. Lukovenkova, Y.V. Marapulets, A.A. Kim et al., Proc. of SCM'19, 58 (2019) 
11. E.L. Afraimovich, G.A. Zherebtsov, N.P. Perevalova et al., Seismoionosfernye $i$ seismoelektromagnitnye protsessy v Baikalskoi riftovoi zone (SB RAS, Novosibirsk, 2012)

The research was supported by Russian Science Foundation (project No. 18-11-00087) 